\title{
Emerging role of extracellular microRNAs and IncRNAs
}

\author{
Geyu Zhou and Xi Chen
}

\begin{abstract}
The discovery of extracellular non-coding RNAs, including microRNAs (miRNAs) and long non-coding RNAs (IncRNAs), has dramatically altered our understanding of the role RNA plays in organisms. Importantly, these extracellular miRNAs and IncRNAs are promising noninvasive biomarkers for diagnosis and prognosis of pathological status. Extracellular vesicles (EVs) are natural carriers of secreted extracellular miRNAs and IncRNAs. Although knowledge regarding the biologically relevant role of extracellular miRNAs is expanding, whether extracellular IncRNAs play a similar role remains incompletely understood. Here, we review the current knowledge regarding extracellular miRNAs and highlight a recent advance demonstrating that extracellular IncRNAs may also mediate cell-to-cell communication and other complicated biological processes.
\end{abstract}

Keywords: Exosome, microRNA, IncRNA

\section{Introduction}

Non-coding RNAs such as microRNAs (miRNAs) and long non-coding RNAs (lncRNAs) are critical regulators of gene expression. MiRNA is typically $19-24 \mathrm{nt}$ in length, processed inside the cell by the enzyme Dicer, incorporated into the RNA-induced silencing complex (RISC) in the single-stranded form, and mediates post-transcriptional silencing through binding to the 3 '-UTR region of target genes [1]. LncRNA is a heterogeneous class of transcripts with a minimum length of $200 \mathrm{nt}$ and limited protein coding potential [2]. Although lncRNA and mRNA are both transcribed by RNA polymerase II, there are many features of IncRNAs that are not found in mRNAs, such as undergoing special 3 '-terminal processing and splicing [3]. Readers may refer to other review for detailed description of lncRNA biogenesis [3]. Although most lncRNAs remain functionally uncharacterized, some have been validated to regulate gene expression via different mechanisms of action, including transcriptional regulation by recruiting protein complexes and post-transcriptional regulation by pairing with other RNAs [4]. Both miRNAs and lncRNAs are associated with various biological processes, such as

\footnotetext{
* Correspondence: xichen@nju.edu.cn

State Key Laboratory of Pharmaceutical Biotechnology, NJU Advanced Institute for Life Sciences (NAILS), Jiangsu Engineering Research Center for MicroRNA Biology and Biotechnology, School of Life Sciences, Nanjing University, 163 Xianlin Avenue, Nanjing 210046, Jiangsu, China
}

development $[5,6]$, differentiation $[7,8]$ and pathogenesis $[9,10]$.

The distribution of miRNAs and lncRNAs is not limited to the intracellular space. The extracellular space is a treasure abundant with not only metabolites, ions, and proteins but also with non-coding RNAs. For example, miRNAs can be robustly detected in body fluids including plasma/serum [11], urine [12], milk [13] and saliva [14]. These miRNAs are stable and resistant to RNase degradation and other harsh extracellular conditions [11]. Similarly, several lncRNAs are present and detectable in plasma [15] and urine [16]. Like intracellular miRNAs and lncRNAs, circulating miRNAs and lncRNAs are also related with disease status and have clinical potential to serve as biomarkers for diagnosis and prognosis of various diseases, such as diabetes [11, 17] and gastric cancer $[15,18]$.

\section{Exosome/microvesicle-mediated intercellular transfer of miRNAs regulates physiological and pathological processes}

Extracellular vesicles (EVs) are a heterogeneous collection of membrane-bound vesicles released by cells that mediate intercellular communication [19]. EVs can be divided into several sub-categories based on their size, cellular origin and biogenesis $[19,20]$. Here, we just focus on two classes of extracellular vesicles - exosomes 
and microvesicles (MVs). Exosomes (40-120 nm) are natural, nanosized intraluminal microvesicles released by cells and present in biological fluids as natural transporters of bioactive molecules [21]. Their formation is driven by sequential formation of early endosome, late endosome/multivesicular bodies (MVBs) and intraluminal vesicles (ILVs), that upon exocytosis are released as exosomes, which is also called the endolysosomal pathway [21, 22]. Microvesicles $(50-1000 \mathrm{~nm})$ are generated by budding from the plasma membrane [20]. Notably, the term 'exosome' and 'microvesicle' are used interchangeably in many published articles due to the incomplete understanding of their biogenesis, inconsistency in purification and lack of thorough characterization protocol [20]. In this article, we also use these two terms interchangeably.

Recent studies have demonstrated that exosomes play an important role in intercellular communication through horizontal transfer of miRNAs [23, 24]. More specifically, subsets of miRNAs can be selectively packaged into exosomes. The sorting process is controlled by complex mechanisms. For example, heterogeneous nuclear ribonucleoprotein A2B1 (hnRNPA2B1) binds exosomal miRNAs through recognition of a specific sequence motif on miRNAs and controls their loading into exosomes [25]. The release of exosomes is also regulated by delicate machinery, involving ceramide sphingolipids, Rab GTPase, SNARE complexes and other critical molecules [21, 26, 27]. Subsequently, the released exosomes carry encapsulated miRNAs between neighbouring and distant cells, where they are ultimately taken up and exert regulatory functions in the recipient cells. Many studies have shown that this type of intercellular communication between various cells can have significant biological functions (Fig. 1).

Secreted extracellular miRNAs are regulators of various physiological processes (Fig. 1a-e). Mittelbrunn et al. found that $\mathrm{CD}^{+}{ }^{+}$exosomes derived from $\mathrm{T}, \mathrm{B}$, and dendritic immune cells contain miRNAs, and these exosomes promote antigen-driven unidirectional transfer

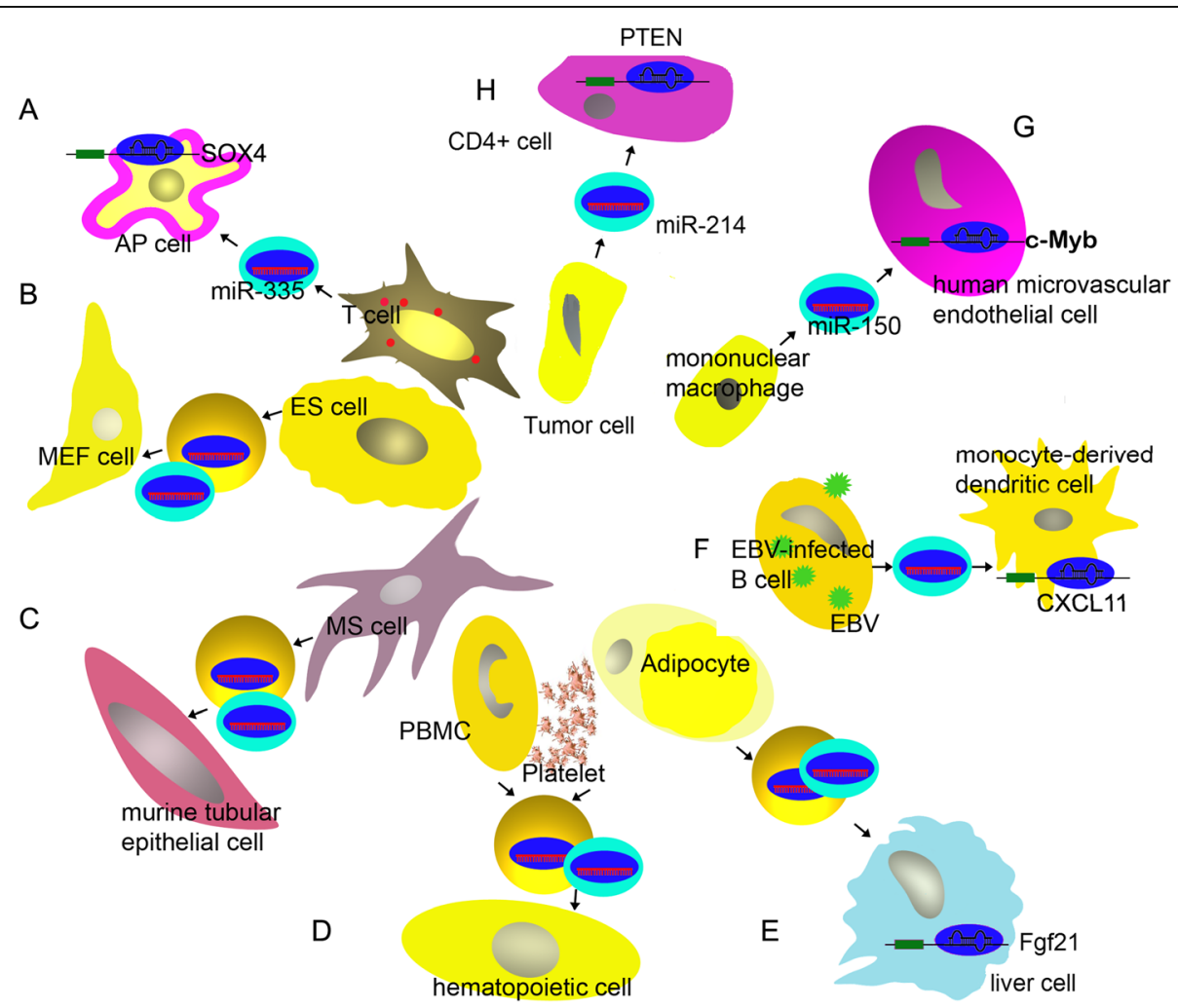

Fig. 1 Schematic description of the roles of secreted miRNAs in various physiological and pathological processes. a miR-335 secreted from T cells was transported via exosomes to recipient antigen-presenting cells and inhibited the translation of SOX4 mRNA. $\mathbf{b}$ miRNAs secreted from embryonic stem cells were transported to recipient mouse embryonic fibroblasts via microvesicles. c miRNAs secreted from mesenchymal stem cells were transported to recipient murine tubular epithelial cells via microvesicles. $\mathbf{d}$ miRNAs secreted from PBMCs and platelets were transported to recipient haematopoietic cells via microvesicles. e miRNAs secreted from adipocytes were transported to recipient hepatocytes via exosomes and downregulated the expression of Fgf21. $\mathbf{f}$ EBV miRNAs secreted by EBV-infected B cells were transported via exosomes to uninfected monocyte-derived dendritic cells and suppressed the translation of CXCL11 mRNA. g miR-150 secreted from the macrophage cell line THP-1 was transported via microvesicles to the recipient microvascular endothelial cell line HMEC-1 and downregulated the expression of c-Myb. h miR-214 secreted from tumour cells was transported via microvesicles to CD4 ${ }^{+}$cells and downregulated the expression of PTEN 
of miRNAs, such as miR-335, from $\mathrm{T}$ cells to antigenpresenting cells (APCs) during T cell-APC cognate immune interactions (Fig. 1a) [28]. They further proposed that this type of miRNA transfer can fine-tune gene expression during generation of the immune response and increase the complexity of communication between immune cells. Yuan et al. demonstrated that microvesicles derived from embryonic stem cells contained abundant miRNAs that can be transferred to mouse embryonic fibroblasts in vitro (Fig. 1b) [29]. In another study, Collino et al. demonstrated that microvesicles released from human bone marrow-derived mesenchymal stem cells and liver resident stem cells contained miRNAs specific to the cell origin (Fig. 1c) [30]. Moreover, they reported that miRNAs from mesenchymal stem cells were transferred to recipient murine tubular epithelial cells via microvesicle. Hunter et al. observed $\sim 100$ miRNAs in plasma microvesicles and found significant differences in miRNA expression among plasma microvesicles, platelets, and peripheral blood mononuclear cells (PBMCs) of healthy donors (Fig. 1d) [31]. Through prediction of the gene targets and biological pathways regulated by miRNAs, they found that the majority of miRNAs expressed in plasma microvesicles might regulate the metabolic homeostasis of haematopoietic cells and haematopoiesis [31]. Adipose tissue is another source of circulating miRNAs. Ogawa et al. found that microvesicles secreted by cultured $3 \mathrm{~T} 3-\mathrm{L} 1$ adipocytes harboured nearly 7000 transcripts and 140 miRNAs and that the abundance of adipocyte-related miRNAs in microvesicles increased as differentiation proceeded [32]. Thomou et al. observed significantly decreased levels of circulating exosomal miRNAs in mice with adipose tissue-specific Dicer knockout and in humans with lipodystrophy. Furthermore, they discovered that adipose-derived exosomal miRNAs are associated with the expression of hepatic and circulating Fgf21 mRNA, as well as glucose tolerance (Fig. 1e) [33]. Thus, it is important to note that extracellular miRNAs secreted from one tissue can regulate gene expression in a distant tissue.

On the other hand, roles for extracellular miRNAs in pathogenesis have also been elucidated (Fig. 1f-h). For example, Epstein-Barr virus (EBV)-transformed B lymphoblastoid cells (B-LCL) were able to secrete exosomal EBV miRNAs, which were transferred to uninfected primary immature monocyte-derived dendritic cells (MODCs) where viral miRNAs repressed the expression of CXCL11, an immunoregulatory gene downregulated in primary EBV-associated lymphomas [34]. A previous study from our group found that microvesicles derived from the plasma of atherosclerosis patients and cultured THP-1 cells had high levels of miR-150, and these microvesicles were taken up by HMEC-1 cells. Exogenous miR-150 suppressed the c-Myb expression and promoted the migration of recipient HMEC-1 cells [24]. Given that the migration of endothelial cells lining blood vessels is a major component of atherosclerosis, elevated miR-150 levels in microvesicles may mediate crosstalk between circulating monocytes/macrophages and vascular endothelial cells under inflammatory conditions (Fig. 1g) [24]. Notably, exosomal oncogenic miRNAs secreted from tumour cells have fundamental roles in tumourigenesis, including remodelling the tumour microenvironment, suppressing immune response, promoting tumour metastasis and disseminating drug resistance [35, 36]. Another study by our group discovered that secreted miR-214 from tumour cells suppressed the expression of PTEN in recipient $\mathrm{CD} 4^{+}$cells, resulting in Treg expansion, enhanced immune suppression and accelerated tumour growth (Fig. 1h) [37].

\section{Exosomes contain extracellular IncRNAs and mediate local horizontal transfer of IncRNAs between tumour cells to disseminate drug resistance}

Compared with the accumulating evidence of intercellular communication mediated by extracellular miRNAs, little is known about whether such horizontal and functional transfer also involves lncRNA. Deep sequencing revealed that IncRNA can also be detected in plasma-derived exosomal RNA $(\sim 5 \%)$ [38]. Like miRNAs, only a subset of IncRNAs are selectively loaded into exosomes, which may be associated with physiological and cellular factors $[39,40]$. Ahadi et al. found that lncRNAs having certain miRNAs seed regions are enriched in prostrate cancer derived exosomes, which may also relate to the high expression level of those miRNAs [40]. The presence of lncRNAs in exosomes also create the opportunity to use them as non-invasive biomarkers for diagnosis of diseases, such as diabetes [17], gastric cancer [15] and colorectal cancer [41]. Furthermore, in vitro studies have shown that extracellular vesicles containing certain lncRNAs can modulate the sensitivity of recipient cells' response to anti-cancer drugs [42, 43]. For example, elevated expression of linc-ROR is associated with TGF-beta induced chemoresistance in HCC cells and enriched in HCC cells derived exosomes. Incubating with these exosomes can increase the expression of lnc-ROR and promote the chemoresistance in recipient cells. Thus, it is interesting to speculate whether lncRNA can be transmitted by exosomes and exert biological functions in vivo.

A recent study by $\mathrm{Qu}$ et al. reports the first case in which one lncRNA (lncARSR) is packaged into exosomes and transferred to recipient cells to disseminate sunitinib resistance (Fig. 2) [44]. The authors first established sunitinib-resistant and sunitinib-sensitive renal cell carcinoma (RCC) cell lines and identified lncARSR 


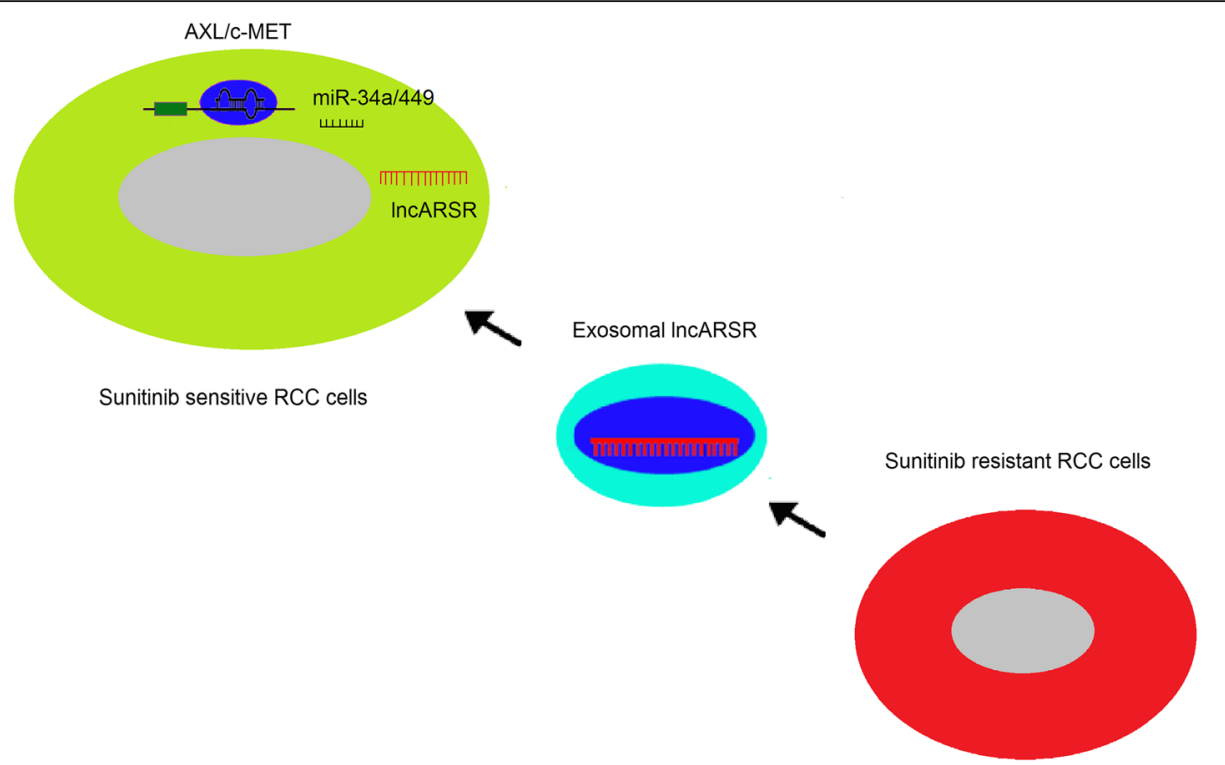

Fig. 2 Schematic diagram of IncARSR transfer mediated by exosomes. IncARSR was transmitted from sunitinib resistant cells to sunitinib sensitive cells and act as endogenous competing RNA for miR-34a and miR-449. The trapping of miR-34a and miR-449 by IncARSR thus increased the expression their targets AXL and c-MET, which is responsible for sunitinib resistance

to be significantly upregulated in sunitinib-resistant cell lines. Furthermore, they showed that the expression levels of lncARSR in plasma and tumour tissues were correlated with the resistance of patients' response to sunitinib treatment.

The authors further investigated whether lncARSR is present in the form of exosomes. They found that lncARSR was enriched in exosomes derived from sunitinib-resistant cell lines. Importantly, the RNA binding protein hnRNPA2B1, which is involved in selective exosomal miRNA trafficking [25], was also responsible for loading lncARSR into exosomes. This is reasonable because lncARSR shares a specific sequence motif near the $5^{\prime}$ end with hnRNPA2B1-recognizable miRNAs. This finding also suggests that selective loading of miRNAs and lncRNAs into exosomes may share common mechanisms. The authors then provided direct evidence that the intercellular transfer of lncARSR was mediated by exosomes. They fluorescently labelled cell-derived exosomes containing FITC-labelled lncARSR with Dil and observed the co-localization of FITC and Dil signals in recipient cells. In contrast, no internalization was observed when incubating naked FITC-lncARSR with recipient cells.

A co-culture colony formation assay revealed that sunitinib-sensitive RCC cells became sunitinib-resistant after incubation with sunitinib-resistant RCC cells. Either reducing the level of lncARSR or inhibiting the expression of molecules responsible for production and secretion of exosomes, such as RAB27A/B, nsMase and hnRNPA2B1, in resistant cells was able to suppress or abolish the competence of phenotypic conversion. In addition, an RCC xenograft model revealed that exosomes derived from sunitinib-resistant but not sunitinib-sensitive cells dampened the response of subcutaneous tumours to sunitinib in vivo. These results confirmed that intercellular

Table 1 Representative studies on exosome/microvesicle-mediated functional intercellular transfer of miRNAs or IncRNAs

\begin{tabular}{|c|c|c|c|c|}
\hline MiRNA/IncRNA & Donor cell & Target cell & Biological function & References \\
\hline miR-335 & T cells & Antigen presenting cells & Regulate immune process & [28] \\
\hline $\begin{array}{l}\text { Adipose-derived exosomal } \\
\text { miRNAs }\end{array}$ & Adipocytes & Liver cells & $\begin{array}{l}\text { Regulate glucose } \\
\text { metabolism }\end{array}$ & [33] \\
\hline EBV miRNAs & EBV infected B cells & Monocyte-derived dendritic cells & $\begin{array}{l}\text { Repress immunostimulatory } \\
\text { gene }\end{array}$ & [34] \\
\hline $\operatorname{miR}-150$ & $\begin{array}{l}\text { Human monocytic cell line } \\
\text { (THP-1) }\end{array}$ & $\begin{array}{l}\text { Human microvascular endothelial cell line } \\
\text { (HMEC-1) }\end{array}$ & Promote cell migration & [24] \\
\hline $\operatorname{miR}-214$ & Tumor cells & $\mathrm{CD}^{+}$cells & Regulate immune response & {$[37]$} \\
\hline IncARSR & Sunitinib resistance RCC cells & Sunitinib sensitive RCC cells & Induce chemoresistance & [44] \\
\hline
\end{tabular}


trafficking of lncARSR exosomes between sunitinib-resistant and sunitinib-sensitive RCC cells was indispensable for the dissemination of sunitinib resistance.

The authors went on to identify how lncARSR contributes to the establishment of sunitinib resistance in RCC cells. They screened multiple receptor tyrosine kinases (RTKs) in resistant cells and found two RTKs, AXL and c-MET, that were decreased after knockdown of lncARSR, which also abolished the established lncARSR-driven sunitinib resistance. Given that lncRNA can function as competing endogenous RNA (ceRNA) to sequester miRNAs and thus disinhibit the expression of miRNA target proteins, the authors proposed that lncARSR might interact with AXL/c-MET-targeted miRNAs to confer sunitinib resistance. Bioinformatic analysis predicted that IncARSR and the 3'-UTR of AXL/c-MET share binding sites for miR-34/ 449. Subsequent RIP and luciferase assays confirmed that miR-34/449 physically and functionally interacted with lncARSR. Similarly, the authors showed that miR-34/449 could inhibit the expression of AXL/c-MET, and conversely, inhibiting miR-34/449 facilitated the expression of AXL/c-MET. As RTKs, AXL/c-MET can activate STAT3, AKT and ERK signalling upon receiving the proper signals. The authors also found that the transcriptional factors FOXO1 and FOXO3a, which are downstream effectors of activated AKT, could bind and suppress the expression of IncARSR, thus forming an overall positive feedback loop between lncARSR and AXL/c-MET.

\section{Concluding remarks}

Extracellular miRNAs and functional exosome-mediated transfer of miRNAs can be viewed as an additional method of intercellular communication. Over the last decade, evidence showing that extracellular miRNAs can have both local and distant biological effects has accumulated. It also appears that this type of communication is common in multiple cell types (Table 1). Moreover, the presence of other extracellular non-coding RNAs, such as lncRNA, in exosomes/microvesicles raises the question of whether these RNAs also carry functional information. The first report that exosomal lncARSR mediates the dissemination of drug resistance in tumour cells interestingly suggests such a possibility. However, there are also many questions that remain unanswered. For example, it is important to investigate whether the intercellular communication mediated by exosomal IncRNAs is indeed a universal phenomenon that occurs in various cell lines. Additionally, the key molecules and mechanisms responsible for biogenesis, sorting, release and uptake of secreted lncRNAs remain incompletely understood. In conclusion, extracellular miRNAs and lncRNAs may play important roles in the intercellular signalling system and further knowledge will trigger more research to understand their physiological and pathological roles.

\section{Abbreviations}

APCs: antigen-presenting cells; B-LCL: B lymphoblastoid cells; ceRNA: competing endogenous RNA; EBV: Epstein-Barr virus; EVs: extracellular vesicles; hnRNPA2B1: heterogeneous nuclear ribonucleoprotein A2B1; ILVs: intraluminal vesicles; IncRNAs: long non-coding RNAs; miRNAs: microRNAs; MODCs: monocyte-derived dendritic cells; MVBs: multivesicular bodies; MVs: microvesicles; PBMCs: peripheral blood mononuclear cells; RCC: renal cell carcinoma; RISC: RNA-induced silencing complex; RTKs: receptor tyrosine kinases

\section{Acknowledgements \\ Not applicable.}

\section{Funding}

This work was supported by grants from the National Natural Science Foundation of China (No. J1103512 and J1210026) and the Natural Science Foundation of Jiangsu Province (No. BE2016737).

Availability of data and materials

Not applicable.

\section{Authors' contributions}

GZ drafted the manuscript. XC contributed to designing and revising the manuscript. They both approved the final manuscript.

Ethics approval and consent to participate

Not applicable.

Consent for publication

Not applicable.

Competing interests

The authors declare that they have no competing interests.

\section{Publisher's Note}

Springer Nature remains neutral with regard to jurisdictional claims in published maps and institutional affiliations.

Received: 5 May 2017 Accepted: 26 February 2019

Published online: 05 March 2019

References

1. Bartel DP. MicroRNAs: genomics, biogenesis, mechanism, and function. Cell. 2004;116(2):281-97.

2. Ulitsky I, Bartel DP. lincRNAs: genomics, evolution, and mechanisms. Cell. 2013;154(1):26-46.

3. Quinn JJ, Chang HY. Unique features of long non-coding RNA biogenesis and function. Nat Rev Genet. 2016;17(1):47-62.

4. Quek XC, Thomson DW, Maag JL, Bartonicek N, Signal B, Clark MB, Gloss BS, Dinger ME. IncRNAdb v2.0: expanding the reference database for functional long noncoding RNAs. Nucleic Acids Res. 2015;43(Database issue):D168-73.

5. Devaux Y, Zangrando J, Schroen B, Creemers EE, Pedrazzini T, Chang CP, Dorn GW 2nd, Thum T, Heymans S. Long noncoding RNAs in cardiac development and ageing. Nat Rev Cardiol. 2015;12(7):415-25.

6. Wu J, Bao J, Kim M, Yuan S, Tang C, Zheng H, Mastick GS, Xu C, Yan W. Two miRNA clusters, miR-34b/c and miR-449, are essential for normal brain development, motile ciliogenesis, and spermatogenesis. Proc Natl Acad Sci U S A. 2014;111(28):E2851-7.

7. Chen ZH, Wang WT, Huang W, Fang K, Sun YM, Liu SR, Luo XQ, Chen YQ. The IncRNA HOTAIRM1 regulates the degradation of PML-RARA oncoprotein and myeloid cell differentiation by enhancing the autophagy pathway. Cell Death Differ. 2017;24(2):212-24.

8. Malpeli G, Barbi S, Zupo S, Tosadori G, Scardoni G, Bertolaso A, Sartoris S, Ugel S, Vicentini C, Fassan M, et al. Identification of microRNAs implicated in the late differentiation stages of normal B cells suggests a central role for miRNA targets ZEB1 and TP53. Oncotarget. 2017:8(7):11809-826. https:// www.ncbi.nlm.nih.gov/pubmed/28107180.

9. Wei S, Wang K. Long noncoding RNAs: pivotal regulators in acute myeloid leukemia. Exp Hematol Oncol. 2015;5:30. 
10. Liu Y, Liu R, Yang F, Cheng R, Chen X, Cui S, Gu Y, Sun W, You C, Liu Z, et al. miR-19a promotes colorectal cancer proliferation and migration by targeting TIA1. Mol Cancer. 2017;16(1):53.

11. Chen X, Ba Y, Ma L, Cai X, Yin Y, Wang K, Guo J, Zhang Y, Chen J, Guo X, et al. Characterization of microRNAs in serum: a novel class of biomarkers for diagnosis of cancer and other diseases. Cell Res. 2008;18(10):997-1006.

12. Stuopelyte K, Daniunaite K, Bakavicius A, Lazutka JR, Jankevicius F, Jarmalaite $\mathrm{S}$. The utility of urine-circulating miRNAs for detection of prostate cancer. $\mathrm{Br}$ J Cancer. 2016;115(6):707-15.

13. Chen X, Gao C, Li H, Huang L, Sun Q, Dong Y, Tian C, Gao S, Dong H, Guan $D$, et al. Identification and characterization of microRNAs in raw milk during different periods of lactation, commercial fluid, and powdered milk products. Cell Res. 2010;20(10):1128-37.

14. Weber JA, Baxter DH, Zhang S, Huang DY, Huang KH, Lee MJ, Galas DJ, Wang K. The microRNA spectrum in 12 body fluids. Clin Chem. 2010;56(11): 1733-41.

15. Arita T, Ichikawa D, Konishi H, Komatsu S, Shiozaki A, Shoda K, Kawaguchi T, Hirajima S, Nagata H, Kubota T, et al. Circulating long non-coding RNAs in plasma of patients with gastric cancer. Anticancer Res. 2013;33(8):3185-93.

16. Lorenzen JM, Schauerte C, Kolling M, Hubner A, Knapp M, Haller H, Thum T. Long noncoding RNAs in urine are detectable and may enable early detection of acute $\mathrm{T}$ cell-mediated rejection of renal allografts. Clin Chem. 2015;61(12):1505-14

17. Carter G, Miladinovic B, Patel AA, Deland L, Mastorides S, Patel NA. Circulating long noncoding RNA GAS5 levels are correlated to prevalence of type 2 diabetes mellitus. BBA Clin. 2015;4:102-7.

18. Liu R, Zhang C, Hu Z, Li G, Wang C, Yang C, Huang D, Chen X, Zhang H, Zhuang $R$, et al. A five-microRNA signature identified from genome-wide serum microRNA expression profiling serves as a fingerprint for gastric cancer diagnosis. Eur J Cancer (Oxford, England : 1990). 2011;47(5):784-91.

19. Maas SL, Breakefield XO, Weaver AM. Extracellular vesicles: unique intercellular delivery vehicles. Trends Cell Biol. 2017;27(3):172-88.

20. El Andaloussi S, Mager I, Breakefield XO, Wood MJA. Extracellular vesicles: biology and emerging therapeutic opportunities. Nat Rev Drug Discov. 2013;12(5):347-57.

21. Kowal J, Tkach M, Thery C. Biogenesis and secretion of exosomes. Curr Opin Cell Biol. 2014;29:116-25.

22. Colombo M, Raposo G, Thery C. Biogenesis, secretion, and intercellular interactions of exosomes and other extracellular vesicles. Annu Rev Cell Dev Biol. 2014:30:255-89.

23. Valadi H, Ekstrom K, Bossios A, Sjostrand M, Lee JJ, Lotvall JO. Exosomemediated transfer of mRNAs and microRNAs is a novel mechanism of genetic exchange between cells. Nat Cell Biol. 2007;9(6):654-9.

24. Zhang Y, Liu D, Chen X, Li J, Li L, Bian Z, Sun F, Lu J, Yin Y, Cai X, et al. Secreted monocytic miR-150 enhances targeted endothelial cell migration. Mol Cell. 2010;39(1):133-44

25. Villarroya-Beltri C, Gutierrez-Vazquez C, Sanchez-Cabo F, Perez-Hernandez D, Vazquez J, Martin-Cofreces N, Martinez-Herrera DJ, Pascual-Montano A, Mittelbrunn M, Sanchez-Madrid F. Sumoylated hnRNPA2B1 controls the sorting of miRNAs into exosomes through binding to specific motifs. Nat Commun. 2013:4:2980.

26. Trajkovic K, Hsu C, Chiantia S, Rajendran L, Wenzel D, Wieland F, Schwille P, Brugger $\mathrm{B}$, Simons $\mathrm{M}$. Ceramide triggers budding of exosome vesicles into multivesicular endosomes. Science. 2008;319(5867):1244-7.

27. Hanson Pl, Cashikar A. Multivesicular body morphogenesis. Annu Rev Cell Dev Biol. 2012;28:337-62.

28. Mittelbrunn M, Gutierrez-Vazquez C, Villarroya-Beltri C, Gonzalez S, SanchezCabo F, Gonzalez MA, Bernad A, Sanchez-Madrid F. Unidirectional transfer of microRNA-loaded exosomes from T cells to antigen-presenting cells. Nat Commun. 2011;2:282.

29. Yuan A, Farber EL, Rapoport AL, Tejada D, Deniskin R, Akhmedov NB, Farber DB. Transfer of microRNAs by embryonic stem cell microvesicles. PLoS One. 2009;4(3):e4722

30. Collino F, Deregibus MC, Bruno S, Sterpone L, Aghemo G, Viltono L, Tetta C, Camussi G. Microvesicles derived from adult human bone marrow and tissue specific mesenchymal stem cells shuttle selected pattern of miRNAs. PLoS One. 2010;5(7):e11803.

31. Hunter MP, Ismail N, Zhang X, Aguda BD, Lee EJ, Yu L, Xiao T, Schafer J, Lee $M L$, Schmittgen TD, et al. Detection of microRNA expression in human peripheral blood microvesicles. PLoS One. 2008;3(11):e3694.
32. Ogawa R, Tanaka C, Sato M, Nagasaki H, Sugimura K, Okumura K, Nakagawa Y, Aoki N. Adipocyte-derived microvesicles contain RNA that is transported into macrophages and might be secreted into blood circulation. Biochem Biophys Res Commun. 2010;398(4):723-9.

33. Thomou T, Mori MA, Dreyfuss JM, Konishi M, Sakaguchi M, Wolfrum C, Rao TN, Winnay JN, Garcia-Martin R, Grinspoon SK, et al. Adipose-derived circulating miRNAs regulate gene expression in other tissues. Nature. 2017; 542(7642):450-5.

34. Pegtel DM, Cosmopoulos K, Thorley-Lawson DA, van Eijndhoven MAJ, Hopmans ES, Lindenberg JL, de Gruijl TD, Wurdinger T, Middeldorp JM. Functional delivery of viral miRNAs via exosomes. P Natl Acad Sci USA. 2010;107(14):6328-33.

35. Zhang L, Zhang S, Yao J, Lowery FJ, Zhang Q, Huang WC, Li P, Li M, Wang $X$, Zhang $C$, et al. Microenvironment-induced PTEN loss by exosomal microRNA primes brain metastasis outgrowth. Nature. 2015:527(7576):100-4

36. Sousa D, Lima RT, Vasconcelos MH. Intercellular transfer of Cancer drug resistance traits by extracellular vesicles. Trends Mol Med. 2015;21(10):595-608.

37. Yin Y, Cai X, Chen X, Liang H, Zhang Y, Li J, Wang Z, Chen X, Zhang W, Yokoyama $\mathrm{S}$, et al. Tumor-secreted miR-214 induces regulatory T cells: a major link between immune evasion and tumor growth. Cell Res. 2014; 24(10):1164-80.

38. Huang $X$, Yuan $T$, Tschannen $M$, Sun Z, Jacob H, Du M, Liang M, Dittmar RL, Liu $Y$, Liang $M$, et al. Characterization of human plasma-derived exosomal RNAs by deep sequencing. BMC Genomics. 2013;14:319.

39. Gezer U, Ozgur E, Cetinkaya M, Isin M, Dalay N. Long non-coding RNAs with low expression levels in cells are enriched in secreted exosomes. Cell Biol Int. 2014;38(9):1076-9.

40. Ahadi A, Brennan S, Kennedy PJ, Hutvagner G, Tran N. Long non-coding RNAs harboring miRNA seed regions are enriched in prostate cancer exosomes. Sci Rep. 2016;6:24922.

41. Liu T, Zhang X, Gao S, Jing F, Yang Y, Du L, Zheng G, Li P, Li C, Wang C. Exosomal long noncoding RNA CRNDE-h as a novel serum-based biomarker for diagnosis and prognosis of colorectal cancer. Oncotarget. 2016;7(51): 85551-63.

42. Takahashi K, Yan IK, Kogure T, Haga H, Patel T. Extracellular vesicle-mediated transfer of long non-coding RNA ROR modulates chemosensitivity in human hepatocellular cancer. FEBS Open Bio. 2014:4:458-67.

43. Takahashi K, Yan IK, Wood J, Haga H, Patel T. Involvement of extracellular vesicle long noncoding RNA (linc-VLDLR) in tumor cell responses to chemotherapy. Mol Cancer Res. 2014;12(10):1377-87.

44. Qu L, Ding J, Chen C, Wu ZJ, Liu B, Gao Y, Chen W, Liu F, Sun W, Li XF, et al. Exosome-transmitted InCARSR promotes Sunitinib resistance in renal Cancer by acting as a competing endogenous RNA. Cancer Cell. 2016;29(5):653-68.

Ready to submit your research? Choose BMC and benefit from:

- fast, convenient online submission

- thorough peer review by experienced researchers in your field

- rapid publication on acceptance

- support for research data, including large and complex data types

- gold Open Access which fosters wider collaboration and increased citations

- maximum visibility for your research: over $100 \mathrm{M}$ website views per year

At $\mathrm{BMC}$, research is always in progress.

Learn more biomedcentral.com/submissions 